\title{
Characterization and development of 56 EST- SSR markers derived from the transcriptome of Odontobutis potamophila
}

\author{
P.P. Wang ${ }^{1,2}$, M. Ren ${ }^{1}$, S.Q. Chen ${ }^{2}$, S.W. Yin ${ }^{1,3}$, C. Zhao ${ }^{1}$, H.Y. Zhang ${ }^{1}$, \\ X.R. Li ${ }^{1}$, Q.Q. $\mathrm{CaO}^{1}$ and G.Q. Zhou ${ }^{2}$ \\ ${ }^{1}$ College of Life Sciences, Key Laboratory of Biodiversity and Biotechnology \\ of Jiangsu Province, Nanjing Normal University, Nanjing, Jiangsu, China \\ ${ }^{2}$ Institute of Fisheries Science of Nanjing city, Nanjing, Jiangsu, China \\ ${ }^{3}$ Co-Innovation Center for Marine Bio-Industry Technology of Jiangsu Province, \\ Lianyungang, Jiangsu, China \\ Corresponding author: S.W. Yin \\ E-mail: yinshaowu@163.com \\ Genet. Mol. Res. 16 (2): gmr16029129 \\ Received September 2, 2016 \\ Accepted March 21, 2017 \\ Published May 10, 2017 \\ DOI http://dx.doi.org/10.4238/gmr16029129
}

Copyright (C) 2017 The Authors. This is an open-access article distributed under the terms of the Creative Commons Attribution ShareAlike (CC BY-SA) 4.0 License.

\begin{abstract}
Expressed sequence tags (ETSs) are the sources of microsatellite development. In this study, we isolated and characterized microsatellite markers for Odontobutis potamophila by using Illumina RNA-sequencing. We sequenced a large number of ESTs and screened 200 potential microsatellites. Consequently, a total of 56 novel polymorphic microsatellite repeat markers were identified in thirty-two individuals from a wild population area (Jiande, Zhejiang Province, China). The number of alleles per locus varied from two to eight, the observed heterozygosity $\left(H_{\mathrm{O}}\right)$ ranged from 0.03571 to 0.9375 , and the expected heterozygosity $\left(H_{\mathrm{E}}\right)$ ranged from 0.14326 to 0.81549 . The average number of alleles, $H_{\mathrm{O}}$, and $H_{\mathrm{E}}$ were 5.0, 0.4467, and 0.5518 , respectively. By the calculation, the range of polymorphism information content (PIC) was 0.1177-0.8492. Most of the loci showed moderate or high polymorphism. These newly developed EST-simple
\end{abstract}

Genetics and Molecular Research 16 (2): gmr16029129 
sequence repeat (EST-SSR) markers would serve as an efficient tool for analyzing population connectivity and provide sufficient information for genetic diversity research, parentage, and molecular breeding of $O$. potamophila and other fishes with similar genetic relationship.

Key words: Odontobutis potamophila; Expressed sequence tags; Microsatellites; RNA sequencing; polymorphism; simple sequence repeat

\section{INTRODUCTION}

Odontobutis potamophila is an important commercial fish species mainly inhabiting China's southeastern rivers, such as a part of the Yangtze River, the Qiantang River, and the Minjiang River systems. This fish is deeply favored by people because of its delicious taste, rich nutrition, and high economic value ( $\mathrm{Li}$ and Liu, 2016). It has become a very promising aquaculture species in China. However, because of the rapidly declining wild fish resources, environmental pollution, overfishing, and growing market demand, O. potamophila has undergone a severe reduction in both size and distribution (Zhu et al., 2014a). In 2012, the International Union for the Conservation of Nature (IUCN) listed O. potamophila as a nearthreatened species (Huckstorf, 2012). Therefore, the aim of our study was to identify and describe novel microsatellite loci in O. potamophila that will be useful for studying the genetic diversity of its wild populations and for exploring genetic markers associated with commercially valuable traits for the aquaculture of this species.

Previous studies have demonstrated that the microsatellite markers derived from expressed sequence tag (ESTs) have achieved high efficiency in gene mapping by EST-simple sequence repeats (EST-SSRs), which are correlated with the genes of known functions and as a useful tool for studying the genetic structure of a fish species (Gao et al., 2012; Hasselman et al., 2013). To this day, several polymorphic microsatellite markers of $O$. potamophila have been developed. However, the molecular markers for $O$. potamophila are still not enough. We cannot fully evaluate the germplasm genetics and molecular-assisted breeding system of this fish. Therefore, we urgently need to develop more molecular markers for this fish.

The denaturing polyacrylamide gel electrophoresis (PAGE) and the capillary electrophoresis (CE) are the two frequently used techniques to estimate the SSR products. Denaturing PAGE does not require expensive instruments and fluorescent labels (Pagel et al., 2016). Comparatively, CE can increase the speed of electrophoresis and provide more accurate data. Therefore, in this study, we developed 56 EST-SSR markers of $O$. potamophila by using the $\mathrm{CE}$ technique, and used these markers to obtain detailed genetic background information about the wild populations of this fish species.

\section{MATERIAL AND METHODS}

Sixty-four healthy experimental fish were collected randomly from Jiande, Zhejiang province, China. We extracted the genomic DNA from their tail fin tissue by using a centrifugal column-type cell/tissue genomic DNA extraction kit (Shanghai Generay Biotech Co., Ltd, Shanghai, China). Thirty-two individuals were randomly selected to screen the EST-SSR markers and the same number of individuals were used for polymorphism analysis. The PRIMER3 software was used to design the primers for polymerase chain reaction (PCR). The microsatellite repeats were screened using the MISA software (MicroSAtellite, http://pgrc.ipk-gatersleben.de/

Genetics and Molecular Research 16 (2): gmr16029129 
misa). Their polymorphism was evaluated by conducting $8 \%(\mathrm{w} / \mathrm{v})$ PAGE. The PCR was carried out in a $10-\mu \mathrm{L}$ reaction mixture containing $50 \mathrm{ng}$ template DNA, $200 \mu \mathrm{M}$ dNTPs, $1500 \mu \mathrm{M}$ $\mathrm{MgCl}_{2}, 10 \mathrm{X}$ PCR buffer, 0.5-1 U Taq DNA polymerase (Shanghai Generay Biotech Co., Ltd, Shanghai, China), $0.04 \mu \mathrm{M}$ M13-tailed forward primer, $0.16 \mu \mathrm{M}$ reverse primer, and $0.16 \mu \mathrm{M}$ fluorescence-labeled M13 primer. The PCR amplification was performed using the following cycling conditions: initial denaturation at $95^{\circ} \mathrm{C}$ for $5 \mathrm{~min}$, followed by 30 cycles of denaturation at $94^{\circ} \mathrm{C}$ for $30 \mathrm{~s}$, annealing at various primer-specific temperatures (Table 1) for $30 \mathrm{~s}$, and extension at $72^{\circ} \mathrm{C}$ for $45 \mathrm{~s}$, followed by 8 cycles of amplification at $94^{\circ} \mathrm{C}$ for $30 \mathrm{~s}$, and a final extension at $72^{\circ} \mathrm{C}$ for 2 min. Subsequently, ABI 3500 XL sequencer (Life Technologies, Foster City, CA, USA) was used to analyze the PCR products, and their sizes were determined using GENEMAPPER, version 4.1. The POPGENE software, version 1.32, was used to calculate the number of alleles per locus, allele size range, observed heterozygosity $\left(H_{\mathrm{O}}\right)$, expected heterozygosity $\left(H_{\mathrm{E}}\right)$, genetic distances, and genetic similarity for each sample. The Hardy-Weinberg equilibrium $(H W E)$ and PIC were estimated by using the programs Arlequin 3.1 and PIC_CALC, respectively. All experiments were accomplished in accordance with the guidelines of the Care and Use of Laboratory Animals protocol in China, and the fish experiment was approved by the Ethics Committee of Experimental Animals at Nanjing Normal University, China.

\begin{tabular}{|c|c|c|c|c|c|c|c|c|c|c|}
\hline Loci & GenBank accession No. & \begin{tabular}{|l|l|} 
Primer sequences $\left(5^{\prime} \rightarrow 3^{\prime}\right)$ \\
\end{tabular} & Repeat motif & $\operatorname{Tm}\left({ }^{\circ} \mathrm{C}\right)$ & Size (bp) & $N_{\mathrm{A}}$ & $H_{\mathrm{O}}$ & $H_{\mathrm{E}}$ & P value HWE & PIC \\
\hline OPRMI & KR861467 & \begin{tabular}{|l|} 
F: GGTGGACGGCAAAGTAAAAA \\
R: TTGCATTTGTAATAAAATTTCTCCC
\end{tabular} & (TTGAA)s & 50 & $138-143$ & 2 & 0.60000 & 0.48814 & 0.26501 & 0.3968 \\
\hline OPRM2 & KR861468 & $\begin{array}{l}\text { F: GGCCCCCTACAGGACATTAG } \\
\text { R: GGTTGTTGCACTTGACCAAAA }\end{array}$ & $($ GATT)s & 50 & $220-254$ & 5 & 0.37500 & 0.68403 & $0.00054^{*}$ & 0.5504 \\
\hline OPRM8 & KR861469 & $\begin{array}{l}\text { F: GGGTCCTTCTCACGGTAACA } \\
\text { R: GAAAAGTTGTGCTGGCTCGT }\end{array}$ & $(\mathrm{T})_{11 \ldots(\mathrm{GAG})_{5}}$ & 50 & $164-176$ & 4 & 0.70370 & 0.72886 & 0.58076 & 0.7693 \\
\hline OPRM10 & KR861470 & $\begin{array}{l}\text { F: ATGTCGAGCAGTAACCAGGG } \\
\text { R: CCGCTCCTTCTGAAAAGAAA }\end{array}$ & $(\mathrm{AT})_{6 \ldots} \ldots\left(\mathrm{CT}_{7}\right.$ & 50 & $292-294$ & 2 & 0.48000 & 0.48980 & 1.00000 & 0.2824 \\
\hline OPRMII & KR861471 & $\begin{array}{l}\text { F: AAACAGGGAAAAGCAAAGCA } \\
\text { R: CCAGGCTTAGGAGAGCAGAA }\end{array}$ & $(\mathrm{TCT})_{5 \ldots} .(\mathrm{T})_{11}$ & 50 & $218-222$ & 3 & 0.44828 & 0.60012 & 0.06964 & 0.7512 \\
\hline OPRM15 & KR861472 & $\begin{array}{l}\text { F: GCTCCAGGTGATGTTGTCCTA } \\
\text { R: CAGGAAGACAGCACTTCAAAAA }\end{array}$ & $(\mathrm{AT})_{7 \ldots} \ldots(\mathrm{TGA})_{5}$ & 50 & $186-196$ & 4 & 0.26667 & 0.27401 & 0.50341 & 0.2322 \\
\hline OPRM17 & KR861473 & $\begin{array}{l}\text { F: CGCCGAACAAACTACTGTCC } \\
\text { R: CTGAAAACAAACCATGGGAAG }\end{array}$ & $(\mathrm{TTA})_{6 \ldots} . .(\mathrm{TTA})_{5}$ & 50 & $244-260$ & 5 & 0.59375 & 0.73462 & 0.15441 & 0.5714 \\
\hline OPRM19 & KR861474 & $\begin{array}{l}\text { F: ATGTCATCGTGGTGGTTTGA } \\
\text { R: AGGGTTTGGAGCAGAGGTT }\end{array}$ & $(\mathrm{TGG})_{s}(\mathrm{TGA})_{s}$ & 50 & $266-278$ & 4 & 0.14815 & 0.14326 & 1.00000 & 0.2970 \\
\hline OPRM23 & KR861475 & $\begin{array}{l}\text { F: ACAAAGCCAACCAACAAAGG } \\
\text { R: GCTAAGGAATTCACCCCACA }\end{array}$ & $(\mathrm{AC})_{7 . \ldots(\mathrm{CA})_{7}}$ & 50 & $280-284$ & 3 & 0.25000 & 0.52179 & 0.01551 & 0.7032 \\
\hline OPRM24 & KR861476 & $\begin{array}{l}\text { F: AACGCCGAACCACAAATACT } \\
\text { R: CCACCTACAGCTCGTGGTTT }\end{array}$ & $(\mathrm{TG})_{6}(\mathrm{~T})_{10}$ & 50 & $236-238$ & 2 & 0.40741 & 0.50664 & 0.43922 & 0.4059 \\
\hline OPRM26 & KR861477 & $\begin{array}{l}\text { F: GATTCCTGGAGAGGGAGGAG } \\
\text { R: ATACCAGCCCAAAATGCGTA }\end{array}$ & $(\mathrm{GT})_{9 . .(\mathrm{TG})_{8}}$ & 50 & $244-264$ & 5 & 0.66667 & 0.79322 & 0.14125 & 0.7273 \\
\hline OPRM30 & KR861478 & $\begin{array}{l}\text { F: AGAGAACAGACTCCAACAGCAA } \\
\text { R: CACCGAACCTGACGATTGAT }\end{array}$ & $(\mathrm{TC})_{9 . . .(\mathrm{CTC})_{5}}$ & 50 & $224-244$ & 6 & 0.50000 & 0.69026 & 0.05474 & 0.5961 \\
\hline OPRM31 & KR861479 & $\begin{array}{l}\text { F: GGCCACATTCTTTTGTTCGT } \\
\text { R: GAGCCTGCCTCTCATTCAAG }\end{array}$ & $(\mathrm{TA})_{6}(\mathrm{~T})_{10}$ & 50 & $248-252$ & 3 & 0.53333 & 0.59492 & 0.12408 & 0.6303 \\
\hline OPRM32 & KR861480 & $\begin{array}{l}\text { F: GGACTCCTGCGACACTGTTT } \\
\text { R: ACGGCCTCCTGTAAATGTTG }\end{array}$ & $(\mathrm{T})_{10 . \ldots}(\mathrm{GT})_{6}$ & 50 & $256-266$ & 3 & 0.58065 & 0.64992 & $0.00080^{*}$ & 0.3945 \\
\hline OPRM38 & KR861481 & $\begin{array}{l}\text { F: CATGCTACTGAGGGCACAGA } \\
\text { R: CGCTTCAAGTTGTCAAATATCG }\end{array}$ & $(\mathrm{T})_{10 \ldots . .(\mathrm{TGA})_{5}}$ & 50 & $282-292$ & 6 & 0.58621 & 0.72232 & 0.02815 & 0.7456 \\
\hline OPRM41 & KR861482 & $\begin{array}{l}\text { F: CACACAATGACAAAGCCCAC } \\
\text { R: TTTCCCCTCATTTTATCCC }\end{array}$ & $(\mathrm{A})_{14 \ldots} .(\mathrm{GT})_{7}$ & 50 & $290-300$ & 4 & 0.50000 & 0.65844 & 0.26196 & 0.4081 \\
\hline OPRM43 & KR861483 & $\begin{array}{l}\text { F: CGTTTATGTTAGGGGGAGGG } \\
\text { R: TCCAGCCTTTATTTTTCCCC }\end{array}$ & $\left(\mathrm{GT}_{6} \ldots . .(\mathrm{GT})_{6}\right.$ & 50 & $284-290$ & 4 & 0.48276 & 0.75257 & 0.01024 & 0.6916 \\
\hline OPRM44 & KR861484 & $\begin{array}{l}\text { F: CCTGTGACATTGTGGCTGTT } \\
\text { R: CATCACAGGCAGGCGTATTA }\end{array}$ & (A) $)_{1 \ldots(\mathrm{TG})_{6}}$ & 50 & $236-258$ & 6 & 0.78125 & 0.76736 & 0.00256 & 0.6156 \\
\hline OPRM45 & KR861485 & $\begin{array}{l}\text { F: TGCCTTGCTCATGTCTGAAG } \\
\text { R: GAAGAGGATCAGTGTTGTGACCT }\end{array}$ & $(\mathrm{T})_{16 \ldots(\mathrm{T})_{14 \ldots} \ldots(\mathrm{AC})_{6}}$ & 50 & $264-287$ & 7 & 0.03571 & 0.78506 & $0.00000^{*}$ & 0.7411 \\
\hline OPRM49 & KT805136 & $\begin{array}{l}\text { F: GTGCCTTGCAGGCTATAGGT } \\
\text { R: CAGAGAATTCCAGGCCATTG }\end{array}$ & $(\mathrm{CA})_{8 \ldots} . .(\mathrm{CA})_{6}$ & 50 & $186-192$ & 3 & 0.16129 & 0.15283 & 1.00000 & 0.5877 \\
\hline OPRM50 & KR861486 & $\begin{array}{l}\text { F: GTGCCTTGCAGGCTATAGGT } \\
\text { R: CAGAGAATTCCAGGCCATTG }\end{array}$ & $(\mathrm{CA})_{8 \ldots} \ldots(\mathrm{CA})_{6}$ & 50 & $185-191$ & 3 & 0.15625 & 0.14831 & 1.00000 & 0.3925 \\
\hline OPRM54 & KR861487 & $\begin{array}{l}\text { F: TTTGCAGAAACGCTCTTGAA } \\
\text { R: CGTTTGATTCATTGAAAGCC }\end{array}$ & $(\mathrm{T})_{11 \ldots} \ldots(\mathrm{TCA})_{6}$ & 50 & $254-260$ & 4 & 0.39286 & 0.66429 & 0.01409 & 0.6094 \\
\hline OPRM55 & KR861488 & $\begin{array}{l}\text { F: TTGCCTTATAAATGCCTTCTTGA } \\
\text { R: GCCACAGTGACACCCTGAAT }\end{array}$ & $(\mathrm{T})_{10}(\mathrm{GA})_{6}$ & 50 & $280-284$ & 2 & 0.34375 & 0.32887 & 1.00000 & 0.4018 \\
\hline OPRM57 & KR861489 & $\begin{array}{l}\text { F: GGGTGTCTACACGACTGCAA } \\
\text { R: GACTCTGATTGTGACGTGGC }\end{array}$ & $(\mathrm{T})_{1 \ldots .}(\mathrm{GA})_{9}$ & 50 & $242-250$ & 3 & 0.26087 & 0.52077 & 0.00976 & 0.4610 \\
\hline OPRM58 & KR861490 & $\begin{array}{l}\text { F: CCCCCTTTTTCTCCACATTT } \\
\text { R: GCACGAGGGAGTTAGCATA }\end{array}$ & (TC) $\ldots . .(\mathrm{CT})_{6}$ & 50 & $222-230$ & 5 & 0.51852 & 0.69811 & 0.01109 & 0.6463 \\
\hline OPRM60 & KR861491 & $\begin{array}{l}\text { F: GGACAAAAGGGCATTAGCAA } \\
\text { R: ACACAACCCTAGCATTTGCC }\end{array}$ & $(\mathrm{TG})_{6 \ldots} \ldots\left(\mathrm{T}_{10}\right.$ & 50 & $206-212$ & 4 & 0.42857 & 0.54091 & 0.16912 & 0.4693 \\
\hline OPRM61 & KR861492 & $\begin{array}{l}\text { F: GGACAAAAGGGCATTAGCAA } \\
\text { R: ACACAACCCTAGCATTTGCC }\end{array}$ & $(\mathrm{TG})_{6 \ldots} \ldots(\mathrm{T})_{10}$ & 50 & 185-187 & 3 & 0.58065 & 0.52459 & $0.00014^{*}$ & 0.4339 \\
\hline OPRM62 & KT805137 & $\begin{array}{l}\text { F: GAGAGGCAGGGAGACAACTG } \\
\text { R: GGTGCCTGTTTTCTGAGAGC }\end{array}$ & $(\mathrm{TG})_{6 \ldots} \ldots(\mathrm{A})_{10}$ & 50 & $182-198$ & 3 & 0.93750 & 0.56548 & $0.00000^{*}$ & 0.5645 \\
\hline
\end{tabular}




\begin{tabular}{|c|c|c|c|c|c|c|c|c|c|c|}
\hline Loci & GenBank accession No. & Primer sequences $\left(5^{\prime} \rightarrow 3^{\prime}\right)$ & Repeat motif & $\operatorname{Tm}\left({ }^{\circ} \mathrm{C}\right)$ & Size (bp) & $N_{\mathrm{A}}$ & $H_{0}$ & $H_{\mathrm{E}}$ & $P$ value HWE & PIC \\
\hline OPRM76 & KT805138 & $\begin{array}{l}\text { F: GCTTTTGAGCCTGCTGTTTT } \\
\text { R: AGGAAGGTGAGGCTGGAAAT }\end{array}$ & $(\mathrm{TA})_{7 \ldots}(\mathrm{GT})_{7}$ & 50 & $272-296$ & 3 & 0.66667 & 0.58156 & 0.01484 & 0.4451 \\
\hline OPRM87 & KR861493 & $\begin{array}{l}\text { F: GACGTCACGGTCAATCAATG } \\
\text { R: CGACACCTGGACAAACAATG }\end{array}$ & $(\mathrm{ATA})_{5}$ & 50 & $270-282$ & 4 & 0.56000 & 0.54531 & 0.51515 & 0.7065 \\
\hline OPRM99 & KT805139 & $\begin{array}{l}\text { F: AGAGGGTTGGGAGGACTGTT } \\
\text { R: AGACCTTAACCCAGCCATC }\end{array}$ & $(\text { TAT })_{2}$ & 50 & $202-206$ & 2 & 0.33333 & 0.40881 & 0.36430 & 0.6220 \\
\hline OPRM104 & KR861494 & $\begin{array}{l}\text { F: GCACTATGTGTTGCTGAGGC } \\
\text { R: CCCTCGTGCTCGTGTCTATT }\end{array}$ & $(\mathrm{ATT})_{6}$ & 50 & $276-285$ & 4 & 0.61290 & 0.73559 & 0.04637 & 0.4042 \\
\hline OPRM109 & KR861495 & $\begin{array}{l}\text { F: TTCTGCCCTCCTTGATGTCT } \\
\text { R: TTGGTTCAAAAGCAGCTGTG }\end{array}$ & (CTT)6 & 50 & $288-292$ & 2 & 0.56667 & 0.46271 & 0.25397 & 0.5666 \\
\hline OPRMI10 & KT805140 & $\begin{array}{l}\text { F: GTTTCAGAATAATGCGCGGT } \\
\text { R: CGTACATGAAGGGAGGTGG }\end{array}$ & $(\mathrm{GCA})_{6}$ & 50 & $202-206$ & 2 & 0.38462 & 0.50679 & 0.25694 & 0.3740 \\
\hline OPRM112 & KR861496 & $\begin{array}{l}\text { F: GGATTTTGGACAACTGGCAC } \\
\text { R: GGCACCTTCTCCAGTCGATAA }\end{array}$ & $($ ATT) 6 & 50 & $226-232$ & 3 & 0.26087 & 0.24058 & 1.00000 & 0.3770 \\
\hline OPRM114 & KR861497 & $\begin{array}{l}\text { F: ATCCCGTGTAACTGTACGGC } \\
\text { R: GTGAGACCACGAGCACTTCA }\end{array}$ & $(\mathrm{GAA})_{6}$ & 50 & $231-248$ & 7 & 0.33333 & 0.75611 & $0.00000^{*}$ & 0.7038 \\
\hline OPRM115 & KR861498 & $\begin{array}{l}\text { F: CTGACAAAAGGCACCAGACA } \\
\text { R: AGGCTGGCCTTGTGACTAAA }\end{array}$ & $(\mathrm{TTG})_{6}$ & 50 & $202-206$ & 2 & 0.48276 & 0.47913 & 1.00000 & 0.4061 \\
\hline OPRM117 & KR861499 & $\begin{array}{l}\text { F: CACAACGCAACCAAAACATC } \\
\text { R: GAGACGCTCCGCATAAACTC }\end{array}$ & $(\mathrm{GAA})_{6}$ & 50 & $272-276$ & 2 & 0.43333 & 0.50339 & 0.48033 & 0.3956 \\
\hline OPRM118 & KT805141 & $\begin{array}{l}\text { F: ACGATGAGGCTCTTTCCAGA } \\
\text { R: CTTGGTGTTGGATTGTCGTG }\end{array}$ & $(\mathrm{GCT})_{6}$ & 50 & $230-236$ & 3 & 0.53125 & 0.59524 & 0.63681 & 0.5813 \\
\hline OPRM120 & KR861500 & $\begin{array}{l}\text { F: AAAGTGAGTGCCAGTCAGCA } \\
\text { R: GCCCTCGCCCTAGTCTTTAC }\end{array}$ & $(\mathrm{TAA})_{6}$ & 50 & $127-172$ & 8 & 0.34483 & 0.81549 & $0.00000^{*}$ & 0.7748 \\
\hline OPRM123 & KR861501 & $\begin{array}{l}\text { F: ACGACAGGCAGCAGAGAAGT } \\
\text { R: ATGGGCTTGGTGTTGTTTC }\end{array}$ & $(\mathrm{CAG})_{6}$ & 50 & $218-227$ & 4 & 0.48276 & 0.67453 & 0.03301 & 0.7685 \\
\hline OPRM124 & KR861502 & $\begin{array}{l}\text { F: :GGAACACACAAGCTCCAAA } \\
\text { R: ACGCGCAGCTATCAATTTCT }\end{array}$ & $(\mathrm{AAT})_{6}$ & 50 & $254-266$ & 3 & 0.12500 & 0.22569 & 0.05065 & 0.1177 \\
\hline OPRM125 & KR861503 & $\begin{array}{l}\text { F: : TGGGAATAACGTCAGGCTTC } \\
\text { R: CACGAGGAGCAATGAATGA }\end{array}$ & $(\mathrm{TCT})_{6}$ & 50 & $160-169$ & 4 & 0.53333 & 0.61864 & 0.11246 & 0.7045 \\
\hline OPRM126 & KR861504 & $\begin{array}{l}\text { F: ACATACCTCTGCATCCCCTT } \\
\text { R: GATCCTGAGTGACCTGAGC }\end{array}$ & $(\mathrm{CTG})_{6}$ & 50 & $118-124$ & 3 & 0.44828 & 0.62674 & 0.03903 & 0.3732 \\
\hline OPRM129 & KR861505 & $\begin{array}{l}\text { F: : TCCCAAAACAAACCCAGAA } \\
\text { R: AAAGGCCGTCTGAGCTGTTA }\end{array}$ & $(\mathrm{CCT})_{6}$ & 50 & $254-266$ & 3 & 0.09375 & 0.14831 & 0.15576 & 0.1719 \\
\hline OPRM151 & KR861506 & $\begin{array}{l}\text { F: : AGTTAGAGGCAACATGGCA } \\
\text { R: CAGTGGGTAAGTTGGGAA }\end{array}$ & $(\mathrm{AG})_{10}$ & 50 & $240-268$ & 5 & 0.06250 & 0.28423 & $0.00003^{*}$ & 0.2678 \\
\hline OPRM154 & KR861507 & $\begin{array}{l}\text { F: TTACAGCCTCCATCAGGGTC } \\
\text { R: TTGTTAAGACCGGGTCCAG }\end{array}$ & $(\mathrm{GT})_{10}$ & 50 & $222-230$ & 3 & 0.61538 & 0.67798 & 0.08565 & 0.8492 \\
\hline OPRM170 & KR861508 & $\begin{array}{l}\text { F: AGCATTCCTGCTGCTGTCTT } \\
\text { R: AACTCCTCTGACTGCCTGGA }\end{array}$ & $(\mathrm{GA})_{9}$ & 50 & $256-272$ & 5 & 0.25000 & 0.53312 & $0.00000^{*}$ & 0.4880 \\
\hline OPRM171 & KR861509 & $\begin{array}{l}\text { F: AGCATTCCTGCTGCTGTCTT } \\
\text { R: AACTCCTCTGACTGCCTGGA }\end{array}$ & $(\mathrm{GA})_{9}$ & 50 & $258-272$ & 4 & 0.44000 & 0.56245 & $0.00001^{*}$ & 0.5001 \\
\hline OPRM176 & KT805142 & $\begin{array}{l}\text { F: AGTCCGACGGAGTTTTTGTG } \\
\text { R: CAATTGACTAGCTGCACGGA }\end{array}$ & $(\mathrm{AC})_{9}$ & 50 & $216-226$ & 6 & 0.61905 & 0.79907 & 0.08677 & 0.4248 \\
\hline OPRM180 & KT805143 & $\begin{array}{l}\text { F: AGCTTCCCAACGTTTACCCT } \\
\text { R: TCAGAGCCAGTGCTGCTTTA }\end{array}$ & $(\mathrm{AC})_{9}$ & 50 & $262-268$ & 4 & 0.50000 & 0.65932 & 0.04475 & 0.5969 \\
\hline OPRM189 & KT805144 & $\begin{array}{l}\text { F: TGTGCAACATCCCTGCTTAG } \\
\text { R: CTCCTTTCTTGCTCTGTGGG }\end{array}$ & $(\mathrm{CA})_{9}$ & 50 & $260-266$ & 4 & 0.90625 & 0.65129 & $0.00052^{*}$ & 0.5813 \\
\hline OPRM191 & KT805145 & $\begin{array}{l}\text { F: CAACCGTAAACCTGTTGCCT } \\
\text { R: CCAACGGAATCCAGCTTAAA }\end{array}$ & (GT) & 50 & $256-264$ & 5 & 0.67742 & 0.69223 & 0.21157 & 0.5734 \\
\hline OPRM195 & KT805146 & $\begin{array}{l}\text { F: TTCTCAGCACATCAACCAGC } \\
\text { R: ATACTGGCATTAGGCAACCG }\end{array}$ & (AC)9 & 50 & $244-252$ & 5 & 0.53571 & 0.61558 & 0.63076 & 0.4698 \\
\hline OPRM197 & KT805147 & $\begin{array}{l}\text { F: TCTAGCAACATTGTGTGCCC } \\
\text { R: CCCCTGACCTTTGATATGGAA }\end{array}$ & $(\mathrm{CA})_{9}$ & 50 & $236-262$ & 8 & 0.33333 & 0.50226 & $0.00049 *$ & 0.6037 \\
\hline OPRM200 & KT805148 & $\begin{array}{l}\text { F: GTGTGTGGAGACAGGACACG } \\
\text { R: AAAAGTATGCACCAGGACG }\end{array}$ & (TG) & 50 & $269-301$ & 6 & 0.16129 & 0.26758 & 0.01903 & 0.3750 \\
\hline
\end{tabular}

Tm, annealing temperature; $N_{\mathrm{A}}$, number of alleles; $H_{\mathrm{O}}$, observed heterozygosity; $H_{\mathrm{E}}$, expected heterozygosity; $\mathrm{P}$ value HWE, probability values for exact tests of Hardy-Weinberg equilibrium (*statistically significant after Bonferroni correction); PIC, polymorphism information content.

\section{RESULTS AND DISCUSSION}

A total of 40,905 EST sequences from the cDNA library of $O$. potamophila were constructed in our laboratory. We identified a total of 1,321 SSR loci, of which 200 were selected for microsatellite marker optimization. All loci were successfully amplified. Finally, fifty-six microsatellites were successfully amplified, and all loci were shown to be polymorphic in the Jiande population. The allele number per locus ranged from two to eight. Expected heterozygosities $\left(H_{\mathrm{E}}\right)$ were $0.14326-0.81549$ (mean 0.5518 ). The $H_{\mathrm{O}}$ level range was $0.03571-0.9375$ (mean 0.4467 ), and the PIC value ranged from 0.1177 to 0.8492 . Three microsatellite loci (OPRM15, OPRM124, and OPRM129) in the Jiande samples showed low polymorphism; all other sites presented moderate or high polymorphism. No significant linkage disequilibrium was observed for any locus in the Jiande samples. Microsatellite loci were successfully investigated for the Jiande population of $O$. potamophila. The repeat motifs, primer sequences, and polymorphic parameters are shown in Table 1.

In the present study, we detected a greater number of polymorphic microsatellite

Genetics and Molecular Research 16 (2): gmr16029129 
markers than the previous studies (Zhang et al., 2014; Zhu et al., 2014b; Li et al., 2015), and most of them were in $H W E$. These newly identified and characterized polymorphic microsatellite loci will serve as a useful tool for determining commercially valuable traits, such as quantitative trait locus position, and for studying population genetic diversity, parentage assessment, and molecular ecology of $O$. potamophila.

\section{Conflicts of interests}

The authors declare no conflict of interest.

\section{ACKNOWLEDGMENTS}

Research supported by the Jiangsu Province Science and Technology Support Program (\#BE2013441), Jiangsu Province Six Talent Peaks of High-Level Talents Project (NY-032), Nanjing Science and Technology Plan Projects (\#201505059), Jiangsu University Brand Professional Construction Project Funds (J1103507), and Project Foundation of the Academic Program Development of Jiangsu Higher Education Institution (PAPD).

\section{REFERENCES}

Gao Z, Luo W, Liu H, Zeng C, et al. (2012). Transcriptome analysis and SSR/SNP markers information of the blunt snout bream (Megalobrama amblycephala). PLoS One 7: e42637. http://dx.doi.org/10.1371/journal.pone.0042637

Hasselman DJ, Ricard D and Bentzen P (2013). Genetic diversity and differentiation in a wide ranging anadromous fish, American shad (Alosa sapidissima), is correlated with latitude. Mol. Ecol. 22: 1558-1573. http://dx.doi.org/10.1111/ $\underline{\text { mec. } 12197}$

Huckstorf V (2012). Odontobutis potamophilus. In: IUCN 2012. IUCN red list of threatened species.

Li Q and Liu Z (2016). New complete mitochondrial genome of the Odontobutis potamophila (Perciformes, Odontobutidae): genome description and phylogenetic performance. Mitochondrial DNA A DNA Mapp Seq. Anal. 27: 163-164.

Li Q, Wang XB and Liu ZZ (2015). Isolation and characterization of polymorphic microsatellite markers for the river sleeper (Odontobutis potamophila). Conserv. Genet. Resour. 7: 251-253. http://dx.doi.org/10.1007/s12686-014-0350-1

Pagel UR, Reis RS, Carvalho VP, Santos EVW, et al. (2016). Comparative analysis of short tandem repeat data obtained by automated and gel electrophoresis techniques. Genet. Mol. Res. 15: 1-7. http://dx.doi.org/10.4238/gmr.15038436

Zhang LJ, Zhang HW, Zhang YP, Zhu F, et al. (2014). Development and characterization of 42 novel polymorphic microsatellite markers for Odontobutis potamophila from EST sequences. Conserv. Genet. Resour. 6: 469-472. http:// dx.doi.org/10.1007/s12686-013-0130-3

Zhu F, Luo J, Yin SW, Zhang LJ, et al. (2014a). Isolation and characterization of twenty-eight polymorphic microsatellite markers in Odontobutis potamophila and cross-amplification in other Gobioidei. Conserv. Genet. Resour. 6: 601-604. http://dx.doi.org/10.1007/s12686-014-0150-7

Zhu F, Zhang LJ, Yin SW, Zhang HW, et al. (2014b). Genetic diversity and variation in wild populations of dark sleeper (Odontobutis potamophila) in China inferred with microsatellite markers. Biochem. Syst. Ecol. 57: 40-47. http:// dx.doi.org/10.1016/j.bse.2014.07.002

Genetics and Molecular Research 16 (2): gmr16029129 